\title{
Optical Excitation of Solid Neon in the Vacuum Ultraviolet*
}

\author{
R. Haensel $\dagger$, G. Keitel†, E. E. Koch \\ Deutsches Elektronen-Synchrotron DESY, Hamburg, Germany
}

(Received 31 August 1970)

\begin{abstract}
The reflection and transmission spectra of solid Ne films have been measured at $6^{\circ} \mathrm{K}$ for photon energies between 10 and $35 \mathrm{eV}$ where transitions from the valence band occur. The use of the continuous spectrum of synchrotron radiation of DESY enabled us to resolve an exciton series with sharp maxima beginning at $17.8 \mathrm{eV}$ and converging to 21.4 $\mathrm{eV}$. At higher energies solid Ne exhibits additional weak structures in the conductionband continuum.
\end{abstract}

In past years excitations from the valence bands of solid argon, krypton, and xenon have been studied optically ${ }^{1-4}$ and by electron-energyloss experiments, ${ }^{5,6}$ but no optical experiments have yet been performed on solid neon. By use of the continuum of synchrotron radiation the valence-band excitations of $\mathrm{Ar}, \mathrm{Kr}$, and Xe were studied recently up to $30 \mathrm{eV}$ with high resolution. ${ }^{2,3}$ In these experiments an efficient reflection technique was used. The improvement of our knowledge of the spectra of the heavier rare gases obtained by these investigations encouraged us to study also solid Ne with the same technique. According to the absorption of gaseous $\mathrm{Ne}^{7,8}$ and a theoretical work on the solid, ${ }^{9} \mathrm{Ne}$ was expected to show its first electronic resonances around $20 \mathrm{eV}$, a region difficult to investigate with high resolution using conventional light sources.

We measured the reflectance of solid $\mathrm{Ne}$ at $6^{\circ} \mathrm{K}$ for an angle of incidence of $15^{\circ}$ in the energy range from 10 to $30 \mathrm{eV}$. The synchrotron radiation of the 7.5-GeV DESY ${ }^{10}$ electron synchrotron was monochromatized by a normal-incidence monochromator in a modified Wadsworth mounting. ${ }^{11}$ The resolution was about $2 \AA$ in wavelength over the whole energy range. The wavelength calibration was accurate to $\pm 2 \AA$. Gaseous Ne (purity $99.99 \%$, purchased from L'Air Liquide) was evaporated onto either a cleaved $\mathrm{KCl}$ single crystal or a polished LiF plate, or a 500 $\AA$ thick $\mathrm{Mg}$ foil, respectively, which were cooled in a He cryostat. The light reflected from the $\mathrm{Ne}$ surface was detected with an open magnetic photomultiplier, Bendix M 306. When a Mg foil was used as a substrate the transmission could be measured simultaneously with the reflection by means of a second magnetic multiplier behind the sample. Cryostat and multipliers were placed in a modified commercial ultrahigh-vacuum system. ${ }^{12}$ After baking the whole system for $8 \mathrm{~h}$ the basic chamber pressure was of the order of $10^{-9}$ Torr. During evaporation oscillatory changes of the intensity of the reflected light, due to interference effects with increasing thickness, were observed for photon energies below the onset of absorption $(16 \mathrm{eV})$. The evaporation was stopped when these oscillations in intensity disappeared, thereby indicating that the films were sufficiently thick to exclude interference. Because of the good vacuum conditions the reflectance remained constant within $10 \%$ over one hour.

Figure 1 shows the reflectance of solid $\mathrm{Ne}$ evaporated onto a $\mathrm{LiF}$ plate or a $\mathrm{KCl}$ single crystal, measured between 16 and $30 \mathrm{eV}$. Below 16 $\mathrm{eV}$ no structure was observed. Above $16 \mathrm{eV}$ the spectrum is dominated by an intense reflectance peak at $17.8 \mathrm{eV}$ followed by a series of weaker maxima at $20.3,20.9$, and $21.3 \mathrm{eV}$, respectively. Between 22 and $30 \mathrm{eV}$ no prominent structures were observed.

The reflectance value for the dominant peak at $17.8 \mathrm{eV}$ was estimated to be $60 \%$ with an er-

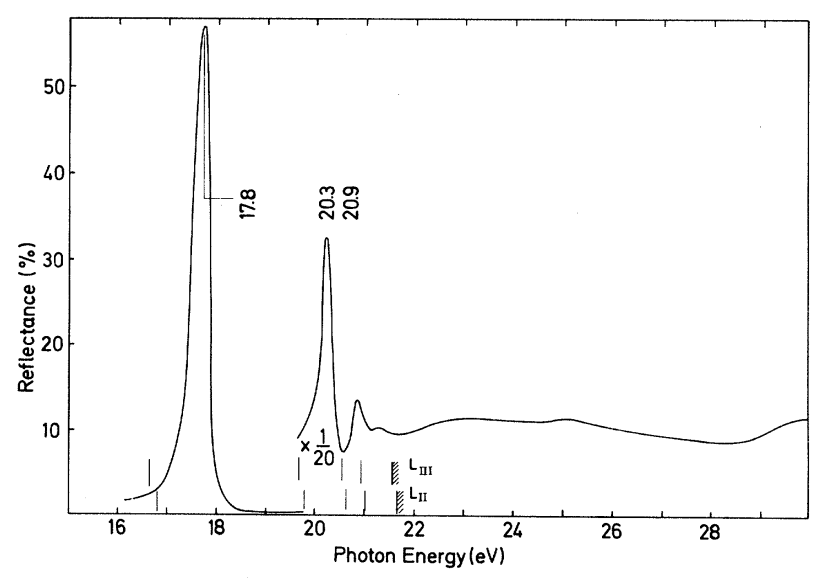

FIG. 1. Reflectance of solid Ne evaporated on a $\mathrm{LiF}$ plate or a $\mathrm{KCl}$ single crystal at $6^{\circ} \mathrm{K}$ (solid line); slit width $2 \AA$. The vertical lines indicate the spin-orbitsplit series $2 p \rightarrow \nu s$ and $2 p \rightarrow \nu s^{\prime}(\nu \geqslant 3)$ with their limits $L_{\text {III }}$ and $L_{\text {II }}$ for gaseous $\mathrm{Ne}^{8}$ 
ror of $\pm 20 \%$ by comparison with the known reflectivity of the $\mathrm{KCl}$ substrate. This first line is about forty times more intense than the second one. The positions of the first three lines agree within $0.1 \mathrm{eV}$ with those found in electron-energy-loss experiments. ${ }^{13,14}$ The fourth line is only resolved in the optical experiment.

In contrast to the other rare gas solids no spin-orbit splitting is observed. The splitting is expected to be close to the value of $0.14 \mathrm{eV}$ found for gaseous $\mathrm{Ne}^{7}$ It should be detectable with the experimental resolution of about 0.05 $\mathrm{eV}$ at $18 \mathrm{eV}$. However, Webber, Rice, and Jortne ${ }^{9}$ predicted a broadening of the lines in the solid and a half-width of the order of $0.4 \mathrm{eV}$. Therefore, we believe that the first line in solid $\mathrm{Ne}$, for which we found a half-width of $0.5 \mathrm{eV}$, consists of the spin-orbit partners broadened so much that the spectrum exhibits only one broad peak.

As for the other rare gas solids one can tentatively ascribe the lines to a Rydberg series, the energies of which are determined by $E_{n}=E_{0}$ $-B n^{-2} \cdot{ }^{15}$ Using the $n=2$ and $n=3$ lines one obtains for the series limit $E_{0}=21.4 \mathrm{eV}$ and the binding energy $B=4.3 \mathrm{eV}$. This value fits quite well into the series of binding energies derived from measurements on the other rare gas sol$\operatorname{ids}^{1-3}: B_{\mathrm{Ar}}=2.3 \mathrm{eV}, B_{\mathrm{Kr}}=1.4 \mathrm{eV}$, and $B_{\mathrm{Xe}}=0.8$ $\mathrm{eV}$. However, a binding energy of $4.3 \mathrm{eV}$ is unusually large for Wannier excitons. Furthermore, in the case of Wannier excitons the line intensities ${ }^{16}$ should vary as $n^{-3}$ whereas we find a much stronger decrease.

On the other hand, one might discuss this part of the solid $\mathrm{Ne}$ spectrum in terms of localized Frenkel excitations. In Fig. 1 the vertical lines indicate the positions of the gas absorption lines for the spin-orbit-split series $2 p \rightarrow \nu s$ and $2 p$ $\rightarrow \nu s^{\prime}(\nu>3)$ with series limits $21.56 \mathrm{eV}$ and 21.7 eV, respectively. ${ }^{8,9}$ This figure shows a close relationship between the line positions in gaseous and solid Ne. For example, one can find a correspondence between all pairs of spin-orbitsplit lines of the gas and the lines of the solid. However, the lines in the solid are shifted to higher energy as compared with the corresponding lines in the gas. This blue shift decreases with increasing quantum number so that the series limit of the gas and that of the solid calculated above with the Rydberg formula $(21.4 \mathrm{eV})$ lie close together. It is interesting to note that the shift $\Delta E$ of the first line in $\mathrm{Ne}$ is $1 \mathrm{eV}$ which is much larger than the corresponding shifts in the

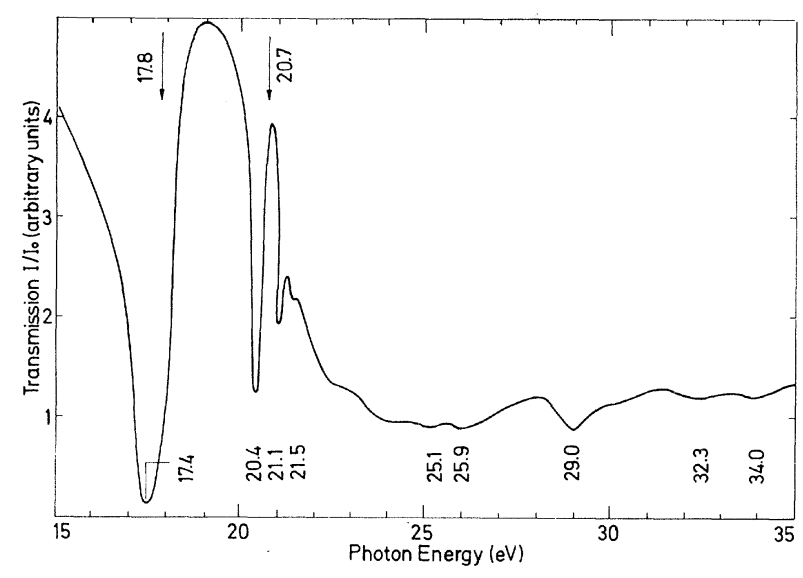

FIG. 2. Transmission of solid Ne films evaporated on $\mathrm{Mg}$ films; slit width $2 \AA$.

other rare gas solids $\left(\Delta E_{\mathrm{Ar}}=0.48 \mathrm{eV}, \Delta E_{\mathrm{Kr}}=0.22\right.$ $\left.\mathrm{eV}, \Delta E_{\mathrm{Xe}}=0.02 \mathrm{eV}\right)$.

We have applied both the Wannier and Frenkel picture in discussing the observed excitations. Neither of the pictures seems to be sufficient, so that we suggest the excitons in solid Ne to be of an intermediate type.

Figure 2 shows the transmission $I / I_{0}$ of solid $\mathrm{Ne}$ between 15 and $35 \mathrm{eV}$ as taken from films evaporated on thin $\mathrm{Mg}$ foils. The arrows indicate the positions of the first two sharp maxima of the corresponding reflection spectrum. ${ }^{17}$ As in the spectrum of Fig. 1, a series of well-resolved sharp minima converging to about $22 \mathrm{eV}$ can be seen. The first minimum at $17.4 \mathrm{eV}$ is very broad and asymmetric. Above the series limit, additional structures were observed. Since no corresponding atomic resonances are known in this region ${ }^{9}$ we ascribe these structures to interband transitions from the $2 p$ valence band to the conduction band. An exact interpretation of these structures will be possible when detailed energyband and density-of-states calculations are available.

We thank P. Schreiber for participation in the early stages of the experiment. We are grateful to H. Boersch, O. Bostanjoglo, L. Schmidt, and P. Krüger for making available to us electronenergy-loss data on solid Ne prior to publication and U. Rössler for prividing us an unpublished Korringa-Kohn-Rostoker band calculation on solid Ne.

*Work supported by the Deutsche Forschungsgemeinschaft. 
$\dagger$ Permanent address: II. Institut für Experimentalphysik der Universität Hamburg, Hamburg, Germany.

\$ Permanent address: Sektion Physik der Universität München, München, Germany.

${ }^{1}$ G. Baldini, Phys. Rev. 128, 1562 (1962).

${ }^{2}$ R. Haensel, G. Keitel, E. E. Koch, M. Skibowski, and P. Schreiber, Phys. Rev. Lett. 23, 1160 (1969).

${ }^{3}$ R. Haensel, G. Keitel, E. E. Koch, M. Skibowski, and P. Schreiber, to be published.

${ }^{4}$ I. T. Steinberger, C. Atluri, and O. Schnepp, J. Chem. Phys. 52, 2723 (1969).

${ }^{5}$ P. Keil, Z. Phys. 214, 251 (1968).

${ }^{6}$ O. Bostanjoglo and L. Schmidt, Phys. Lett. $\underline{22}, 130$ (1966).

${ }^{7}$ C. E. Moore, Atomic Energy Levels, National Bureau of Standards Circular No. 467 (U.S.G.P.O., Washington, D. C., 1949), Vol. I.

${ }^{8}$ K. Codling, R. P. Madden, and D. L. Ederer, Phys. Rev. 155, 26 (1967).

${ }^{9}$ S. Webber, S. A. Rice, and J. Jortner, J. Chem.
Phys. 41, 2911 (1964).

${ }^{10}$ R. Haensel and C. Kunz, Z. Angew. Phys. 23, 276 (1967).

${ }^{11}$ M. Skibowski and W. Steinmann, J. Opt. Soc. Amer. 57, 112 (1967).

${ }^{12} \mathrm{~B}$. Feuerbacher, R. P. Godwin, and M. Skibowski, Rev. Sci, Instrum. 40, 305 (1969)。

${ }^{13} \mathrm{H}$. Boersch, O. Bostanjoglo, and L. Schmidt, "Tagung für Elektronenmikroskopie, Aachen, W. Germany, 1965" (unpublished).

${ }^{14}$ P. Krüger, Diplomarbeit, Universität Hamburg, 1970 .

${ }^{15}$ R. S. Knox, Theory of Excitons (Academic, New York, 1963), Chap. IV.

${ }^{16}$ R. J. Elliott, Phys. Rev. 108, 1384 (1957).

${ }^{17}$ An additional broad maximum has been found between the first two resonances in the corresponding reflection spectrum. This effect is not yet understood, but could be due to a possible hexagonal phase of $\mathrm{Ne}$ instead of the commonly adopted fcc structure.

\title{
Giant Temperature Dependence of Photoemission from the Silver Halides*
}

\author{
R. S. Bauer $\dagger$ and W. E. Spicer \\ Stanford Electronics Laboratories, Stanford University, Stanford, California 94305
}

(Received 23 May 1970)

\begin{abstract}
Anamalous temperature-dependent photoemission has been measured from the silver halides. Structure in the photoelectron energy distributions sharpens dramatically as samples are cooled from 295 to $80^{\circ} \mathrm{K}$. We believe this is due to a lattice vibrationally dependent hybridization of the halogen $p$ and silver $d$ valence states. Using this effect, we have experimentally located the Ag states with almost pure $4 d$ symmetry at 3.7 and 3.3 $\mathrm{eV}$ below the highest valence states in $\mathrm{AgBr}$ and $\mathrm{AgCl}$, respectively.
\end{abstract}

We have observed (see Figs. 1 and 2) unusually large and dramatic changes in the energy distribution curves (EDC's) of electrons photoemitted from the silver halides, upon cooling from 295 to $80^{\circ} \mathrm{K} .^{1}$ The optical properties also exhibit striking, but less well-defined, changes upon temperature variation ${ }^{2,3}$; however, these effects have not been explained previously.

The samples studied were epitaxial, thin films evaporated onto vacuum heat-cleaned silver at less than $3 \times 10^{-8}$ Torr from $99.999 \%$ pure $\mathrm{AgBr}$ and $\mathrm{AgCl}$ powder. The films were about $200 \AA$ thick to eliminate charging at low temperatures. At room temperature, the photoemissive properties of these thin films were essentially identical to those of films $0.6 \mu \mathrm{m}$ thick. Electron microscopy $^{4}$ and optical ${ }^{3,5}$ studies have established that evaporated thin films are good model sys tems for bulk silver halide properties. Photoemission from these samples was measured in situ at less than $5 \times 10^{-11}$ Torr using the standard ac method $^{6}$ for photon energies, $h \nu$, through
$11.8 \mathrm{eV}$. New techniques were developed to measure photoemission at any temperature between room and liquid-nitrogen values. By using forming gas and helium in a liquid-nitrogen heat exchanger, the sample temperature could be set and maintained to within $\pm 4^{\circ} \mathrm{K}$. The experimental results were reproducible following temperature cycling and among different samples.

The most striking feature of the EDC's in the $10-\mathrm{eV}$ photon energy range (see Fig. 1) is the sharpening of the structure with reduced temperature. For example, in $\mathrm{AgBr}$, the full width of the central peak at $90 \%$ of its maximum height decreases from about 0.6 to $0.3 \mathrm{eV}$ upon cooling from 287 to $80^{\circ} \mathrm{K}$. In both materials, room-temperature shoulders are resolved into well-defined peaks at $80^{\circ} \mathrm{K}$. The higher photon energy AgBr EDC's, shown in Fig. 2, exhibit a strong new peak at $80^{\circ} \mathrm{K}$ which is not present at room temperature. As seen in Fig. 1, these changes occur gradually as the temperature is varied.

These changes upon cooling are much larger 